Revue internationale de l'économie sociale

Recma

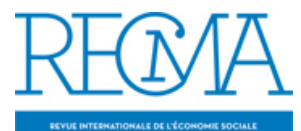

\title{
En bref
}

\section{Marie Graingeot et Patricia Toucas-Truyen}

Numéro 337, juillet 2015

URI : https://id.erudit.org/iderudit/1032518ar

DOI : https://doi.org/10.7202/1032518ar

Aller au sommaire du numéro

\section{Éditeur(s)}

Association Recma

ISSN

1626-1682 (imprimé)

2261-2599 (numérique)

Découvrir la revue

Citer ce document

Graingeot, M. \& Toucas-Truyen, P. (2015). En bref. Revue internationale de

l'économie sociale, (337), 16-22. https://doi.org/10.7202/1032518ar d'utilisation que vous pouvez consulter en ligne.

https://apropos.erudit.org/fr/usagers/politique-dutilisation/ 


\section{EN BREF}

\section{L’économie sociale}

\section{Plan gouvernemental pour}

l'économie sociale au Québec

En mai dernier, le gouvernement du Québec a annoncé, par la voix de son ministre de l'Economie, Jacques Daoust, le lancement de son plan d'action pour l'économie sociale. Le communiqué détaille ainsi les enjeux du secteur dans la région: " $L e$ Québec compte environ 7000 entreprises d'économie sociale. Elles procurent un emploi à plus de 150000 personnes, ce qui représente un peu moins de $4 \%$ de l'emploi total au Québec. Le nombre d'emplois au sein des entreprises d'économie sociale est comparable au niveau d'emploi observé dans des secteurs comme le commerce de gros ou les services aux entreprises. En 2002, le chiffre d'affaires annuel des entreprises d'économie sociale était de 17 milliards de dollars. Aujourd'hui, uniquement pour les entreprises constituées en coopérative ou en mutuelle, ce chiffre atteint 33,4 milliards de dollars. Les résultats attendus sont ambitieux et précis. Par ce plan d'action quinquennal, le gouvernement investit plus de 100 millions de dollars dans le développement de l'économie sociale québécoise. Les mesures qu'il met en ouvre contribueront à créer ou à maintenir 30000 emplois et engendreront des investissements totaux de plus de 500 millions de dollars au cours des cinq prochaines années. "

Plus précisément, les mesures envisagées viseront six objectifs:

- "outiller les entreprises d'économie sociale à toutes les étapes de leur développement; - améliorer l'accès aux marchés et innoveren économie sociale;

- valoriser la réponse des entreprises d'économie sociale aux défis du vieillissement démographique;

- encourager l'insertion socioprofessionnelle au sein des entreprises d'économie sociale;
- soutenir l'entrepreneuriat collectif comme solution à la reprise des entreprises;

- contribuer au développement des entreprises d'économie sociale sur tout le territoire.»

\section{Une charte des circuits courts}

Le 21 avril dernier, Carole Delga, alors secrétaire d'Etat chargée de l'ESS ${ }^{(1)}$, a signé une charte des circuits courts économiques et solidaires (CCES). Ce texte, élaboré par le Labo de l'ESS, vise à mieux définir les CCES : "Un circuit court économique et solidaire est une forme d'échange économique valorisant le lien social, la coopération, la transparence et l'équité entre les acteurs de l'échange." En diffusant cette charte, ses initiateurs espèrent "réunir une communauté d'acteurs » qui partagent ces valeurs afin de "mettre en lumière leur engagement " et de " participer au développement des CCES ». Ainsi, chaque signataire s'engage à :

- "s'investir dans la mise en place d'actions communes, à travers des réalisations pratiques et des processus d'évaluation partagés et contribuer au développement des initiatives existantes;

- être des vecteurs d'information et de communication, mais aussi de collecte et d'échange de bonnes pratiques, notamment en contribuant au développement d'outils; - s'inscrire dans une démarche de capitalisation d'expériences et de mesure de leurs différentes valeurs ajoutées, afin de mutualiser les savoir-faire et les moyens et, ainsi, révéler et renforcer le maillage territorial. " Ces engagements induisent notamment le "développement de partenariats de recherche, l'évolution des politiques publiques et, à terme, la constitution d'un observatoire».

(1) Elle a depuis été remplacée par Martine Pinville. 
Vers une loi sur les monnaies complémentaires?

Une mission d'étude sur les monnaies locales complémentaires et les systèmes d'échange locaux menée par Jean-Philippe Magnen et Nicolas Meunier a remis son rapport à Carole Delga, alors encore secrétaire d'Etat chargée de l'ESS, en avril dernier. Le texte, favorable au développement de ce type de monnaie, propose d'en encourager le développement par la création d'un observatoire, la production d'un guide pratique et le soutien à des initiatives innovantes comme les plateformes régionales autofinancées.

Ce rapport tombe au moment où le Conseil économique, social et environnemental (Cese), qui s'est autosaisi de la question, a rendu un avis sur les « nouvelles monnaies ». Comme le rapporte le site Localtis, «le Cese répertorie [...] quelque 500 monnaies de ce type, au sein desquelles il différencie les monnaies numériques (type Bitcoin), les monnaies locales complémentaires, comme Sol-Violette, et les titres de paiement pouvant par extension être assimilés à des monnaies complémentaires (Air Miles ou Tickets-restaurant) ». Si la masse monétaire représentée par ces monnaies reste faible (10 millions d'euros), leur nombre est en forte progression - elles sont passées de 200 à 500 ces cinq dernières années, dont 30 monnaies locales identifiées par le Cese.

Au sujet des monnaies locales, si la loi de juillet 2014 sur l'ESS leur a donné un cadre légal, celui-ci reste insuffisant pour le Cese, qui relève plusieurs types de risque, notamment le taux de conversion de ces monnaies et la traçabilité des opérations. L’organisme plaide donc pour la création d'un statut juridique "pour l'ensemble des concepts et des systèmes de monnaies complémentaires. [...] Ce statut préciserait les règles de base à respecter en matière de gouvernance, de transparence et de gestion des risques. Il inclurait la nomination d'un responsable, en cas de tromperie, de fraude ou de détournement de fonds, qui devrait être enregistré auprès d'une autorité de contrôle». Ces recommandations incluent la mise en place d'un cadre légal international de régulation, ainsi que l'implication des collectivités territoriales dans la mise en place des systèmes de garantie.

\section{Naissance d'une nouvelle Cress}

L'Aquitaine a désormais sa chambre régionale de l'économie sociale et solidaire (Cress), dont l'assemblée générale constitutive s'est tenue le 31 mars dernier à Bègle. Comme le détaille le communiqué de presse diffusé pour l'occasion, "cette assemblée s'est déroulée au terme de dix-huit mois d'une démarche territoriale de fond visant à la mobilisation autour du projet et à sa préfiguration. Avec le soutien du Conseil national des Cress, du conseil régional d'Aquitaine, de l'Etat, du conseil général de la Gironde, du conseil général des Pyrénées Atlantiques, de la Mutualitéfrançaise, de la Macif et de la Maif, l'association Aquitaine Préfi-Cress, portée par un collectif d'acteurs régionaux de l'économie sociale et solidaire, a élaboré les statuts, le règlement intérieur, le plan d'action et le projet de budget prévisionnel de la Cress Aquitaine, qui ont été adoptés par l'assemblée générale». Jean-Louis Cabrespines, président du Conseil national des Cress, s'est félicité de pouvoir désormais «afficher un réseau complet, pleinement opérationnel, couvrant l'ensemble du territoire ». Le président de cette nouvelle chambre, qui sera opérationnelle à partir de septembre 2015, est Arnaud Virrion, directeur de Arcins environnement service, une structure associative d'insertion par l'activité économique.

\section{Un service d'accompagnement social pour les acteurs de l'ESS $^{\prime}$}

La mutuelle Chorum a lancé en avril dernier Chorum facilit', le premier service d'accompagnement social des acteurs de l'ESS. Cette initiative fait suite au constat dressé dans le Baromètre sur la qualité de vie au travail dans l'ESS établi par la mutuelle en 2014. 
Un site Internet (Chorum-facilit.fr) et une permanence téléphonique offrent aux salariés conseils et accompagnement dans les domaines de la santé, du logement, de la vie familiale et professionnelle. D'après la mutuelle, "près d'un salarié sur deux déclare que ses préoccupations personnelles impactent son travail. A titre d'exemple, l'absentéisme lié à une situation d'aidant peut être évaluéà quinze jours par an. Dans ce contexte, il apparaît nécessaire pour les entreprises de répondre aux besoins sociaux de salariés confrontés à des situations difficiles (décès, handicap, difficultéde santé, précarité...) ou à des moments importants de la vie (arrivée d'un enfant, reconversion professionnelle...).»

Alors que les dispositifs d'aide sociale sont sous-utilisés faute d'être suffisamment connus, Chorum facilit'ambitionne de devenir le "guichet unique de l'accompagnement social ».

\section{La coopération}

\section{Un fonds pour la coopération africaine}

L'Alliance coopérative internationale (ACI) et le Crédit coopératif se sont associés pour relancer le fonds Coopérative de développement internationale, désormais appelé fonds Impact coopératif mondial. Il sera géré par Impulse Europe, filiale belge du Crédit coopératif. Selon Pierre Valentin, directeur général adjoint du Crédit coopératif, ce fonds "répond à la nécessitéd'offrir une facilité de crédit fiable et rentable pour les investissements à long terme dans les coopératives africaines. Le nouveau fonds marque le début d'une ère d'investissements coopératifs et conférera aux coopératives un statut d'actifs financiers à part entière». Il sera notamment associé avec l'Organisation des Nations unies pour l'alimentation et l'agriculture (FAO).

Le site de l'ACI précise que "le fonds a été conçu pour les institutions financières coopératives, telles que les banques coopératives, les coopératives de crédit, les institutions monétaires coopératives ou encore les grandes coopératives dont les activités sont essentiellement tournées vers l'agriculture. En tant que gestionnaire du fonds, Impulse Europe mesurera l'impact social de ses activités d'après des indicateurs de performances clés que recommande Global Impact Investing Network (GIIN) ». La présidente de l'ACI, Pauline Green, a déclaré : "Le nouveau fonds Impact coopératif mondial évaluera la performance non seulement à un niveau financier et social, mais aussi en termes de questions environnementales et de bonne gouvernance. Les bénéficiaires $d u$ fonds devront respecter des normes participatives, éthiques et financières, selon les principes coopératifs. »

\section{Les coopératives colombiennes défendent leur modèle}

En Colombie, les coopératives se sont mobilisées pour faire reconnaître leur modèle par le gouvernement, en riposte à la présentation d'un plan national pour le développement qui autoriserait la Direction nationale de la santé à publier des décrets pour transformer des coopératives en corporations. Comme le rapporte le site de l'ACI, la mesure a finalement été abandonnée, à la satisfaction de Confecoop, la confédération des coopératives colombiennes. Son président, Dario Castillo, a profité de l'occasion pour réclamer la création d'un fonds national pour l'économie sociale, notamment en faveur de la coopération rurale. "Le gouvernement doit comprendre qu'il a devant lui un secteur coopératifdéveloppéqui peut aider le pays. Il est primordial que le modèle d'entreprise de l'économie solidaire soit sans équivoque ", a-t-il déclaré.

\section{La fondation Crédit coopératif fête ses 30 ans}

Les trois décennies d'histoire de la Fondation Crédit coopératif en font l'une des doyennes des fondations d'entreprise en France. 
Dans un livre édité pour l'occasion (2), ses débuts sont ainsi rappelés : "C'est le 18 septembre 1984 que la Fondation du Crédit coopératif - qui perdra l'article au cours des années 2000, pour devenir Fondation Crédit coopératif-voit officiellement le jour. Il faut ici insister sur son caractère pionnier : il n'existe alors aucune loi encadrant les fondations d'entreprise ni aucune règlementation fiscale avantageuse, et le Crédit coopératif compte parmi les premières entreprises à se doterd'une tellefondation. » L'ouvrage retrace les rôles majeurs joués par Jacques Moreau et André Chomel, à l'époque respectivement président et directeur général du Crédit coopératif. Ce dernier deviendra le premier président de la fondation. Parmi les premiers partenariats mis en place par la fondation, nous retiendrons particulièrement le soutien à l'Association pour le développement de la documentation en économie sociale (Addes) ainsi qu'à la Recma, alors encore nommée la Rec (Revue des études coopératives), et qui connaîtra sous la direction d'André Chomel une véritable renaissance.

Pour ce qui est de l'histoire plus récente, Jean-Claude Detilleux, qui présida la fondation de 2004 à avril 2015, rappelle dans la préface de l'ouvrage que, "au cours de ces dernières années, la fondation a pris une nouvelle dimension en accord avec la déclaration de principes du groupe, et son budget a doublé en 2008 en relation avec la croissance de l'activité du Crédit coopératif. Depuis lors, la fondation a connu un dynamisme et un rayonnement remarquables. " Les soutiens qu'elle apporte s'orientent aujourd'hui selon trois axes principaux: la connaissance et reconnaissance de l'ESS, la lutte contre l'exclusion et l'accès à la citoyenneté des personnes handicapées. Elle organise également tous les ans le concours Prix et trophée de l'initiative en économie sociale.

(2) Une Fondation singulière : 1984-2014, Jean-Pierre Mongarny et Eric Belouet, Fondation Crédit coopératif, 2015.

\section{Le succès des Scop ne se dément pas}

La Confédération générale des Scop a publié le 3 juin dernier son bilan chiffré de l'année 2014. Il en ressort que 51000 salariés sont concernés, dont 47500 travaillant dans les sociétés coopératives et participatives (Scop) et 3300 dans les sociétés coopératives d'intérêt collectif (Scic). En 2014, 2800 emplois ont été créés, dont 1940 dans des coopératives nouvellement établies. "En 2014, 277 sociétés coopératives et participatives ont vu le jour. Si les créations ex nihilo restent majoritaires, on assiste à un record en matière de transmissions saines: $20 \%$ des Scop créées l'an passé sont issues d'une transmission", a déclaré Patrick Lenancker, président de la CGscop. Gageons que l'année 2015 ne démentira pas cette tendance. L'actualité a en effet récemment donné un exemple remarqué de reprise d'une entreprise par ses salariés: les anciens de Fralib ont relancé la production de leur usine de thés et d'infusions en mai dernier sous les marques 1336 (comme le nombre de jours de lutte pour créer leur coopérative) et Scop-TI. Le président de la République, François Hollande, s'est lui-même déplacé pour saluer leur succès.

L'expérience pourrait bien faire des émules, par exemple du côté de l'usine Goodyear d'Amiens-Nord, où les salariés défendent actuellement un projet de reprise en Scop.

\section{Les associations}

\section{La famille de l'Udes s'agrandit}

La fédération nationale Familles rurales a rejoint l'Union des employeurs de l'économie sociale (Udes) en mars dernier. Cette fédération est le premier mouvement familial en France et, d'après les chiffres publiés sur son site Internet, regroupe 2300 associations couvrant 10000 communes. Avec 94 fédérations régionales, départementales et nationale, 40000 bénévoles et 20000 salariés, son réseau défend les intérêts de 180000 ménages adhérents dans les domaines des services à la personne, de l'accueil de la petite enfance, de la consommation, des loisirs, etc. 


\section{La qualité de l'emploi}

\section{dans le milieu associatif}

En mars dernier, le Mouvement associatif, qui fédère 600000 associations, a publié une note sur la qualité de l'emploi dans le milieu associatif. Après avoir fait le constat de la croissance continue du secteur $(1,8$ million d'employés à ce jour, soit trois fois plus en trente ans), le document liste les enjeux liés à la qualité de l'emploi : la satisfaction et le bien-être pour les travailleurs, la qualité du service rendu aux usagers, l'attractivité des emplois et les taux d'activité et d'emploi. Le Mouvement associatif caractérise également les différentes dimensions de cette notion: les conditions d'emploi, celles de travail, le dialogue social et la représentation collective, l'accès à la formation tout au long de la vie, l'égalité et la prévention des discriminations et, enfin, la conciliation des temps de vie. Le document rappelle aussi que les conditions d'emploi sont très disparates selon les associations : on observe des conditions salariales très variables et une forte présence des emplois atypiques. Le taux de satisfaction des salariés est néanmoins plus élevé que dans les autres secteurs, du fait notamment de la variété des tâches, de la responsabilité et du sentiment d'utilité, de la possibilité de formation, des inégalités salariales moindres et d'un dialogue social plus dynamique.

Au rang des risques pesant sur la qualité des emplois, le Mouvement associatif compte: le raccourcissement des durées de convention avec les pouvoirs publics ; l'augmentation des besoins sociaux alors que les budgets des pouvoirs publics se contractent et donc la nécessité pour les associations de baisser leurs coûts ; la concurrence accrue avec les entreprises lucratives ; l'idéologie new public management et les logiques gestionnaires entraînant des conflits de valeurs pour les salariés; et, enfin, les mouvements de regroupements et de fusions.

Le document se conclut sur la présentation des "leviers d'amélioration " pour faire face à ces menaces : "développer des outils de suivi de la qualité de l'emploi dans les associations ; inciter les associations à faire vivre en interne des débats sur les critères de la qualité de l'emploi ; valoriser et encourager les bonnes pratiques; impliquer le monde associatif dans les réflexions contemporaines sur la reconfiguration $d u$ travail et son organisation, en favorisant l'innovation dans les métiers et en articulant les trajectoires professionnelles des salariés, celles des bénéficiaires et des organisations. »

\section{Une circulaire sur les relations pouvoirs publics-associations}

Le Mouvement associatif nous informe que le gouvernement est en train de préparer une nouvelle circulaire, dite Valls, encadrant les relations entre associations et pouvoirs publics dans la continuité de la charte des engagements réciproques signée le 14 février 2014 : "Cette circulaire remplacera la circulaire dite Fillon datant du 18 janvier 2010 et précisera le cadre d'attribution des subventions. [...] Elle est marquée par la co-construction et le partenariat. La capacité d'innovation des associations y est largement reconnue et le critère de l'initiative est réaffirmé comme un élément majeur de distinction entre commande publique et subvention. L'augmentation du recours à la commande publique et les appels à projets trop encadrés y sont présentés comme des sources d'affaiblissement de la capacité d'innovation des associations. "

Le Mouvement associatif ayant été associé aux travaux préparatoires de cette circulaire en salue le texte provisoire, le jugeant " plutôt satisfaisant ». Il annonce également que la publication de la circulaire sera suivie de celle d'un "guide pratique d'usage de la subvention en direction des collectivités territoriales».

Marie Graingeot

\section{Les mutuelles}

\section{Du grain à moudre pour le congrès de la FNMF}

Le $41^{\mathrm{e}}$ congrès de la Fédération nationale de la Mutualité française (FNMF) s'est déroulé 
du 11 au 13 juin à Nantes, en présence de plus de 2000 mutualistes. C'est au sein de ces congrès triennaux que, depuis cent vingtdeux ans ${ }^{(3)}$, les responsables des mutuelles françaises définissent les grandes orientations stratégiques du mouvement.

Le thème du congrès 2015, «Utilité sociale, justice et efficacité: une ambition pour les entreprises mutualistes ", rend clairement compte de la double exigence qui, depuis toujours, contraint l'action mutualiste: concilier le social et l'économique. Ce que l'intitulé du congrès ne dit pas, mais que chacun lira en creux, c'est que les principes liminaux du modèle mutualiste - solidarité et égalité de traitement entre les membres, autodétermination par la voie démocratique - n'entrent pas vraiment en résonnance avec les mesures qui lui seront prochainement appliquées (infirmant par la même le dernier principe énoncé).

Voici ce qui attend les mutuelles santé:

- l'ordonnance de transposition dans le droit français de la directive européenne Solvabilité II est parue au Journal officiel du 3 avril. Si les dispositions relatives au durcissement des règles prudentielles n'entreront en vigueur qu'en janvier 2016, celles qui sont relatives à la gouvernance sont immédiatement applicables. Chaque organisme mutualiste doit désigner deux dirigeants effectifs; la responsabilité des administrateurs est renforcée, ce qui suppose que ceux-ci soient d'abord choisis sur la base de leurs compétences, puis formés dans les domaines sensibles de la gestion, du droit etc. Ensuite, le directeur général, désigné comme "dirigeant opérationnel » ne sera plus nommé par le président, mais par le conseil d'administration. Une première lecture de cette mesure autorise un point de vue optimiste: il semblerait que Solvabilité II insufflerait plus de transparence dans la gouvernance mutualiste et que la fonction d'administrateur soit mieux

(3) Le premier congrès mutualiste a été organisé à Lyon en 1883, dix-neuf ans avant la création de la FNMF. valorisée dans le parcours personnel du militant. D'un point de vue plus circonspect, cet engagement sur la voie de la professionnalisation des élus porte le risque d'un affadissement de la fonction politique de ces derniers, voire d'une confusion des genres entre administrateurs et salariés, entre président et directeur. La tendance impulsée par les directives européennes se poursuit, rétrécissant la marge de manœuvre des organismes au nom de l'efficacité économique, négligeant la qualité de mouvement social qui caractérise la mutualité en France depuis le XIX ${ }^{\mathrm{e}}$ siècle;

- la généralisation de la complémentaire santé à tous les salariés du secteur privé, en application de l'accord national interprofessionnel (ANI) signé en février 2013 entre les organisations patronales et les trois syndicats CFDT, CFTC et CFE-CGC. Cette mesure pourrait constituer un réel progrès social si elle concernait tous les citoyens, sans discrimination, mais, en l'état, elle contribue surtout à accentuer les inégalités, écartant les autres catégories de travailleurs (secteur public, professions indépendantes) et ceux qui sont insérés sur le marché du travail (chômeurs de longue durée, jeunes en situation de précarité, retraités).

Bien que ne bénéficiant qu'à quelque 400000 salariés non pourvus d'une complémentaire santé d'entreprise, cette disposition devrait avoir un effet non négligeable sur le fonctionnement des mutuelles, qui se sont historiquement construites sur le principe de l'adhésion volontaire individuelle. On observe d'ores et déjà une accélération de la compression du secteur des mutuelles santé, incitées depuis plusieurs années à mettre en commun leurs ressources de toutes natures (financières, humaines, informatiques...) afin d'être économiquement plus performantes. En témoignent les rapprochements récents de la MGEN et d'Harmonie Mutuelle ou encore de Malakoff Médéric et de la Mutuelle générale. L'enjeu pour ces mutuelles qui, même prises séparément, occupaient déjà le haut 
du pavé mutualiste est de proposer aux employeurs concernés des contrats collectifs suffisamment attractifs sur ce terrain très concurrentiel.

Le monde mutualiste est plus que jamais à la croisée des chemins et les débats ont été animés lors du congrès de Nantes, tant entre les représentants mutualistes et les décideurs institutionnels et politiques, qu'entre les mutualistes eux-mêmes.

\section{L'expertise mutualiste est trop peu sollicitée}

Dénonçant sans relâche le rôle de "payeur aveugle » que l'Etat tend à lui faire jouer depuis des années, la FNMF a émis des propositions pour rendre plus solidaire le fonctionnement de l'Assurance maladie, notamment: - réduire significativement la taxe spéciale sur les conventions d'assurance (TSCA) sur les contrats santé. De fait, si la TSCA, payée par les complémentaires santé, permet à $85 \%$ de financer la couverture maladie universelle (CMU) ${ }^{(4)}$, elle a pour effet paradoxal d'augmenter le tarif des cotisations qui pèsent lourdement sur les adhérents les plus vulnérables. Ainsi, selon l'Institut de recherche et documentation en économie de la santé (Irdes), le nombre des personnes ayant renoncé à contracter une complémentaire santé est passé de 2,8 millions en 2010 à 3,3 millions en 2012 ;

- étendre les réseaux de soins (actuellement limités aux spécialistes) à tous les professionnels de santé afin de faire baisser le coût des restes à charge pour les ménages.

Les pouvoirs publics seraient bien inspirés de prêter une oreille plus attentive aux propositions et aux mises en garde émanant de l'institution mutualiste pour améliorer le fonctionnement général du système de santé et contenir la hausse des dépenses de l'Assurance maladie. Cela éviterait peut-être des mesures contre-productives, à l'instar du contrat d'accès aux soins (CAS) mis en place en décembre 2012 pour limiter le montant des dépassements d'honoraires médicaux et qui a finalement abouti à ce que davantage de praticiens s'adonnent à cette pratique. Moins élevés en moyenne, mais plus nombreux, les dépassements d'honoraires coûtent au total plus cher à la collectivité ${ }^{(5)}$.

\section{La LMDE perd la délégation de gestion du régime étudiant de Sécurité sociale}

Après plusieurs mois d'incertitude, le sort de La Mutuelle des étudiants (LMDE) a finalement été tranché. La mutuelle étudiante connaissait des difficultés financières récurrentes depuis sa création sur les cendres de la MNEF en 2000. Elle sera désormais adossée à la Caisse nationale d'assurance maladie des travailleurs salariés (Cnamts) pour le régime de Sécurité sociale des étudiants. Cette décision, qui sonne la fin de la délégation octroyée à la MNEF dès sa fondation en 1948, devrait permettre aux quelque 920000 adhérents de la LMDE d'être remboursés plus rapidement de leurs frais médicaux. La LMDE continuera à assurer les fonctions d'affiliation et de prévention, ainsi que la gestion de la complémentaire santé, jusqu'à ce que celle-ci trouve un repreneur, puisqu'elle est à vendre.

PATRICIA TOUCAS-TRuyen 